\title{
Définition d'indices successionnels pour la caractérisation du processus de la succession post-culturale au Burundi
}

Frédéric BANGIRINAMA ${ }^{1,5}$ Marie José BIGENDAKo ${ }^{2}$ Jean LEJOLY ${ }^{3}$ Nausicaa NoRET ${ }^{4}$ Charles De CANNIÈre ${ }^{5}$ Jan BOGAERT ${ }^{5}$

${ }^{1}$ École normale supérieure BP 6983, Bujumbura Burundi

${ }^{2}$ Université du Burundi BP 2700, Bujumbura Burundi

${ }^{3}$ Herbarium et Bibliothèque de botanique africaine Université libre de Bruxelles CP 169, avenue F. D. Roosevelt 50 1050 Bruxelles

Belgique

${ }^{4}$ Laboratoire d’écologie végétale et de biogéochimie

Université libre de Bruxelles CP244, boulevard du Triomphe 1050 Bruxelles

Belgique

${ }^{5}$ Laboratoire d'écologie du paysage et systèmes de production végétale Université libre de Bruxelles École interfacultaire de bioingénieurs CP 169, avenue F. D. Roosevelt 50 1050 Bruxelles



Faciès à Cassia occidentalis dans les jeunes jachères de Rusizi (palmeraie). Photo F. Bangirinama. 


\section{RÉSUMÉ}

\section{DÉFINITION D’INDICES SUCCESSIONNELS POUR LA CARACTÉRISATION DU PROCESSUS DE LA SUCCESSION POST-CULTURALE AU BURUNDI}

Des recherches ont été menées sur la biodiversité des jachères situées dans la périphérie des aires protégées du Burundi. Les données relatives aux traits biologiques (forme de vie, types de diaspores et types foliaires) ont été collectées sur divers compartiments reflétant les facteurs spatial et temporel. Les résultats montrent des variations significatives de certains attributs des traits biologiques au cours de la dynamique post-culturale. Parmi trois indices définis pour caractériser cette variabilité, l'efficacité de deux indices (indice successionnel de forme de vie et indice successionnel de dispersion) s'est révélée significative, l'autre (indice successionnel de type foliaire) étant plus lié aux caractéristiques du site. Cependant, des disparités régionales liées à l'effet du facteur climatique ont été mises en évidence.

Mots-clés : indice successionnel, traits biologiques, jachère, restauration, Burundi.

\section{ABSTRACT}

\section{DEFINITION OF SUCCESSIONAL INDEXES TO CHARACTERISE THE POST-CULTIVATION SUCCESSION PROCESS IN BURUNDI}

Research was carried out on biodiversity in fallow lands surrounding protected areas in Burundi. Data on biological traits (life forms, seed dispersal and leaf size) were collected for the different compartments, reflecting relevant spatial and temporal factors. Results show significant variations in some attributes of these biological traits during post-cultivation dynamics. We determined three indexes to characterize this variability. Two of these were significantly effective (Successional Index of life form and Successional Index of seed dispersal), while the third (Successional Index of leaf size) was more correlated to site features. Regional disparities linked to the climatic factor effect were also brought to light.

Keywords: successional indexes, biological traits, fallows, restoration, Burundi.

\section{RESUMEN}

\section{DEFINICIÓN DE ÍNDICES SUCESIONALES \\ PARA LA CARACTERIZACIÓN DEL PROCESO DE LA SUCESIÓN POSTCULTIVO EN BURUNDI}

Se han llevado a cabo investigaciones sobre la diversidad de los barbechos situados en la periferia de las áreas protegidas de Burundi. Los datos de los rasgos biológicos (forma de vida, tipos de dispersión y tipos foliares) han sido cochezados en diferentes compartimentos que reflejaban los dos factores espacial y temporal. Los resultados muestran variaciones significativas de algunos atributos de los rasgos biológicos durante la dinámica postcultivo. Se definieron tres índices para caracterizar dicha variabilidad. La eficacia de dos de estos tres índices (indice sucesional de formas de vida e índice sucesional de dispersión) resulta ser significativa, el otro (índice sucesional de tipo foliar) está más bien vinculado a las características del sitio. Sin embargo, se evidenciaron disparidades regionales con relación al efecto del factor climático.

Palabras clave: índices sucesionales, rasgos biológicos, barbechos, restauración, Burundi. 


\section{Introduction}

La recolonisation naturelle progressive intéresse non seulement les forestiers, pour la gestion et l'aménagement des territoires, mais aussi les scientifiques qui souhaitent mieux connaître la structure, le fonctionnement et la dynamique des communautés végétales au cours de la succession secondaire. Cette étude de la caractérisation des différents stades successionnels des jachères du Burundi cherche à établir, à partir des traits biologiques de la tige, des diaspores et des feuilles, un outil opérationnel en écologie de la restauration.

En effet, les diaspores, les feuilles et la tige constituent les parties de la plante facilement observables sans sa destruction. Les premières assurent la dissémination et la pérennité de l'espèce, les secondes constituent le moteur, par la photosynthèse et la respiration, du fonctionnement de la plante entière. Enfin, la tige est le squelette dont la taille traduit la vitesse de croissance des individus d'une espèce.

Partant de ces observations, Théophraste (372-287 av. J.C.), cité par TESTI (2008), a proposé la première classification des végétaux en supposant des comportements différents selon leur forme de croissance (FC) (herbacées, arbustes, arbres). RAUNKIAER (1934) proposa une classification originale des plantes selon les formes de vie (FV), restée au centre des études en écologie fonctionnelle et basée sur la position des bourgeons végétatifs par rapport à la surface du sol.
RAUNKIAER (1934) proposa en outre une nouvelle classification fondée sur la surface du limbe. Cette dernière est plus liée à la capacité de photosynthèse de la plante. DANSEREAU et LEMS (1957) ont, quant à eux, retenu une classification selon les modes de dissémination.

De nombreuses études ont traité l'importance de ces caractéristiques des plantes dans la compréhension des stratégies adaptatives (MAC ARTHUR, WILSON, 1967 ; GRIME, 1974, 1979, 2001 ; WeStOBY et al., 2002) et des processus physiologiques (CHAPIN, 1993 ; SANTIAGO, WRIGHT, 2007).

À partir de données brutes collectées, BANGIRINAMA et al. (2009 b) ont montré que les spectres de ces traits morphologiques des plantes constituent de bons indicateurs de la succession post-culturale. Toutefois, il est apparu que leur utilisation garde un caractère local. L'objectif de la présente étude est d'intégrer ces indicateurs pertinents au sein d'une seule variable (indice successionnel) ayant un champ d'application plus large. Nous définissons ainsi trois indices successionnels originaux et testons leur efficacité quant au suivi du processus de la succession post-culturale.

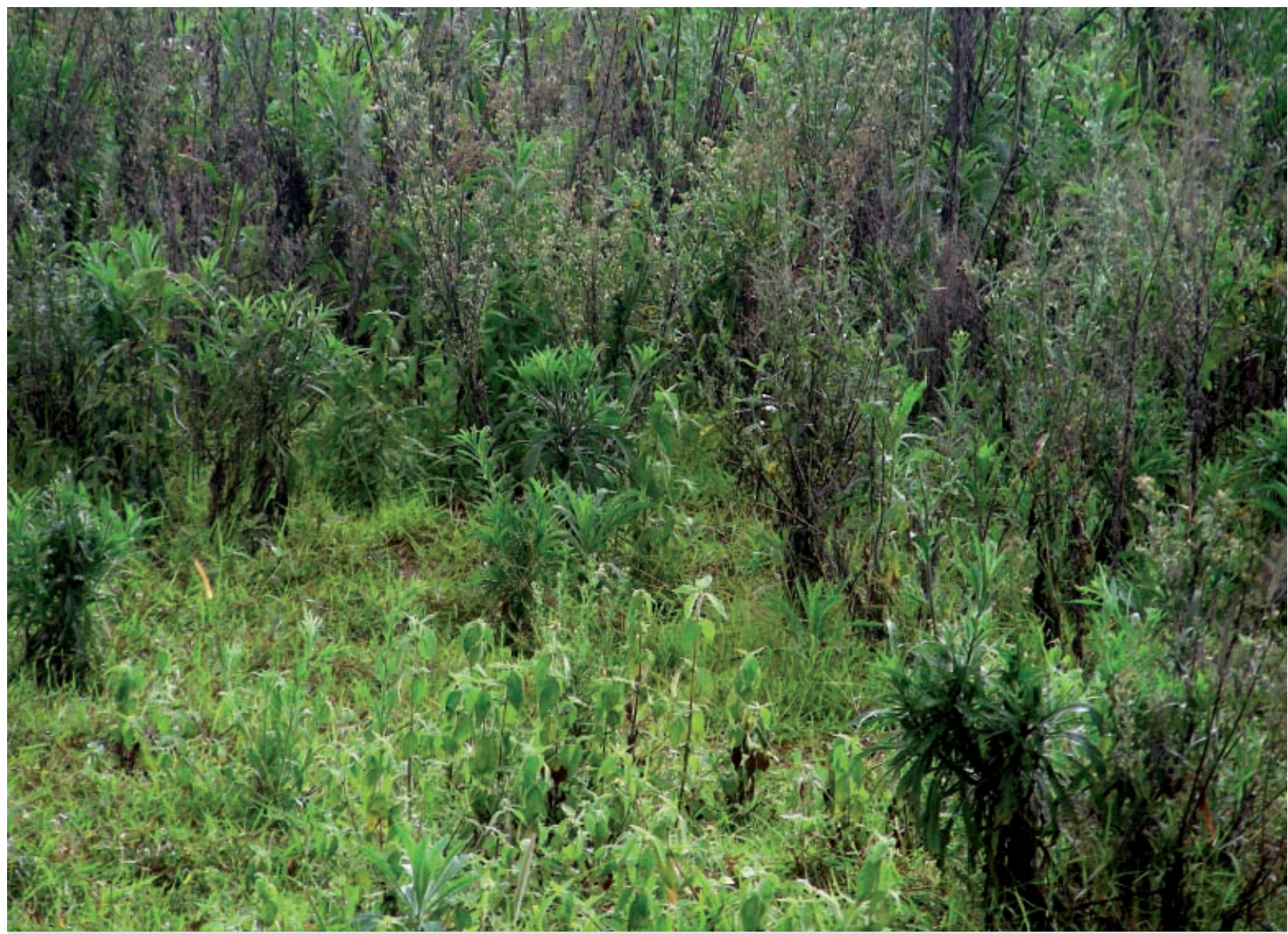

Faciès à Conyza bonariensis dans les jeunes jachères de Ruvubu. Photo F. Bangirinama. 


\section{Méthodes}

\section{Sites d'étude}

Cette étude a été effectuée dans cinq aires protégées du Burundi (réserves naturelles forestières de Kigwena et de Bururi, parc national de la Ruvubu et réserve naturelle de la Rusizi) choisies en considérant les districts phytogéographiques du Burundi. La réserve naturelle de la Rusizi a été subdivisée en deux sites, soit cinq sites au total (figure 1).

La réserve naturelle de la Rusizi, localisée dans le district du graben occidental, est établie à $800 \mathrm{~m}$ d'altitude sur des sols alluvionnaires de la plaine de la Rusizi. Elle est divisée en deux secteurs séparés : le secteur « delta » autour de l'embouchure de la Rusizi et le secteur "palmeraie » au nord. Avec $1000 \mathrm{~mm}$ de précipitations moyennes annuelles, la région présente comme végétation des bosquets xérophiles à Cadaba farinosa et Hyphaene petersiana et des forêts sclérophylles à Euphorbia candelabrum et Grewia mollis.

La réserve naturelle forestière de Kigwena est située à $800 \mathrm{~m}$ d'altitude dans le prolongement sud-ouest du district du Mosso et de la Malagarazi. Sa proximité avec le lac Tanganyika lui confère un climat tropical humide (1 200 mm de pluie par an). La végétation est une forêt dense à Newtonia buchananii et Albizia zygia.

La réserve naturelle forestière de Bururi est située dans le district afro-montagnard, sur les hauteurs de la crête Congo-Nil à plus de 1900 m d'altitude. Les précipitations abondantes (1 $500 \mathrm{~mm}$ par an) entretiennent une forêt ombrophile de montagne à Entandrophragma excelsum et Prunus africana.
Enfin, le parc national de la Ruvubu est situé dans le district du Rwanda-Burundi, sur les plateaux centraux à 1500 m d'altitude. Les précipitations avoisinent 1300 mm par an. La végétation est dominée par des savanes arborées à Parinari curatellifolia et Hyparrhenia div. sp.

\section{Collecte des données}

L'approche synchronique a été retenue pour cette étude de la succession post-culturale. Les relevés phytosociologiques ont été effectués sur plusieurs types de jachères réparties en trois classes d'âge (0-2 ans, 3-5 ans et plus de 5 ans) compte tenu de la rareté des stades plus avancés. L'aire minimale des placettes adoptée dans cette étude découle de résultats de nos travaux préliminaires (BANGIRINAmA et al., 2009 a). Elle a été estimée autour de $32 \mathrm{~m}^{2}$ dans les jachères de $0-2$ ans, de $128 \mathrm{~m}^{2}$ dans les jachères de 3-5 ans et de $64 \mathrm{~m}^{2}$ dans les jachères de plus de 5 ans. Les photos ci-dessous montrent la physionomie de quelques jachères échantillonnées.

Dans chaque site, 10 relevés ont été effectués dans chaque classe d'âge de jachère, soit 30 relevés par site et 150 relevés au total. Pour chaque relevé, il a été inventorié toutes les espèces présentes et estimé leur abondancedominance selon l'échelle de BRAUN-BLANQUET (1932) (tableau I). Après identification des spécimens, la nomenclature adoptée pour les différents taxons a été celle de LEBRUN et STORK (1991-1997). À chaque espèce récoltée, il a été affecté les catégories de traits biologiques.

Les traits biologiques retenus sont indiqués ci-après.

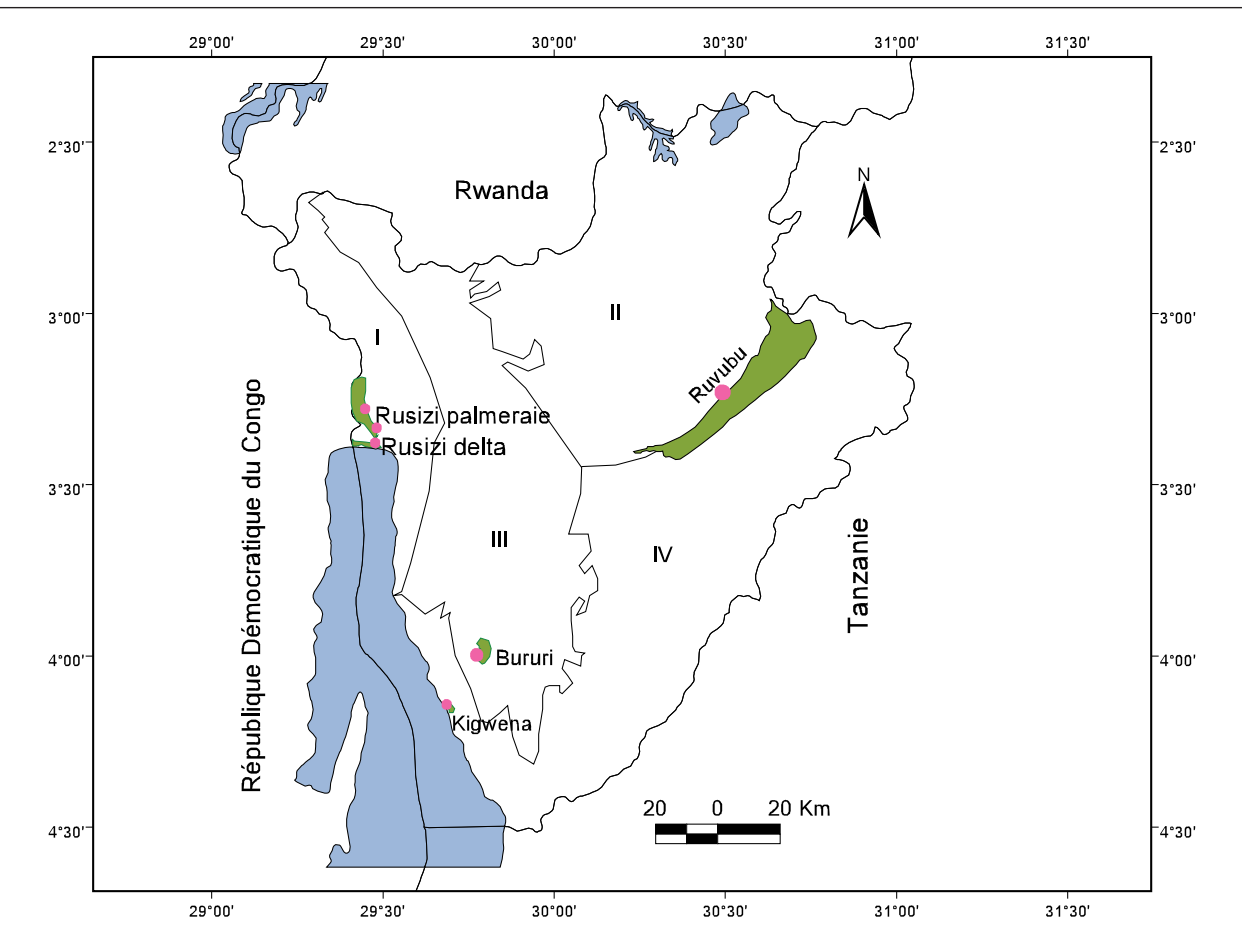

Figure 1.

Localisation des stations échantillonnées dans les districts phytogéographiques du Burundi (I : district du Graben occidental, II : district du Rwanda-Burundi, III : district afro-montagnard et IV : district du Mosso et de la Malagarazi).
Les types biologiques, liés à l'état phénologique de l'espèce rencontrée, ont été utilisés suivant le système de RAUNKIAER (1934) modifié par LEBRUN (1947) :

- phanérophytes : plantes dont l'appareil caulinaire porte à plus de $40 \mathrm{~cm}$ du sol des bourgeons persistants visibles ;

- chaméphytes : plante ayant un appareil végétatif nain, inférieur à $40 \mathrm{~cm}$, avec des bourgeons persistants protégés par des débris de plantes ;

- hémicryptophytes : plantes dont les pousses ou bourgeons de remplacement sont situés au niveau du sol ;

- thérophytes : plantes annuelles qui passent la mauvaise saison sous forme de graines;

- géophytes : plantes possédant un appareil caulinaire caduc dont les bourgeons et les jeunes pousses se trouvent dans le sol. 
Tableau I.

Coefficients d'abondance-dominance de BRAUN-BLANQUET (1932) et recouvrements moyens respectifs.

\begin{tabular}{|c|c|c} 
Coefficient & $\begin{array}{c}\text { Recouvrement } \\
\text { de l'espèce (\%) }\end{array}$ & $\begin{array}{c}\text { Recouvrement } \\
\text { moyen (rec. moy.) (\%) }\end{array}$ \\
\hline 5 & 75 à 100 & 87,5 \\
\hline 4 & 50 à 75 & 62,5 \\
\hline 3 & 25 à 50 & 37,5 \\
\hline 2 & 5 à 25 & 15 \\
\hline 1 & 1 à 5 & 3 \\
\hline & $<1$ & 0,5 \\
\hline
\end{tabular}

Les types de diaspores ont été définis selon la classification de Dansereau et Lems (1957):

- autochores :

- ballochores : diaspores éjectées par la plante elle-même ;

- barochores : diaspores caractérisées par leur poids et l'absence d'une autre caractéristique en rapport avec la dispersion ;

- hydrochores : diaspores ayant un dispositif de flottaison (pléochores) ;

- anémochores :

- pogonochores : diaspores à appendices plumeux ou soyeux, poils et aigrettes ;

- ptérochores : diaspores munies d'appendices ailés ;

- sarcochores : diaspores pourvues de couches externes charnues et molles;

- zoochores :

- sclérochores : diaspores non charnues, relativement légères;

- desmochores: diaspores accrochantes ou adhésives, déhiscentes ou non.

Les types foliaires sont issus de la classification de RAUNKIAER (1934):

- aphylle : pas de feuilles ;

- leptophylle : surface foliaire inférieure à $0,2 \mathrm{~cm}^{2}$;

- nanophylle : surface foliaire comprise entre 0,2 et $2 \mathrm{~cm}^{2}$;

- microphylle : surface foliaire comprise entre $2 \mathrm{~cm}^{2}$ et $20 \mathrm{~cm}^{2}$;

- mésophylle : surface foliaire comprise entre $20 \mathrm{~cm}^{2}$ et $200 \mathrm{~cm}^{2}$;

- macrophylle : surface foliaire comprise entre $2 \mathrm{dm}^{2}$ et $20 \mathrm{dm}^{2}$;

- mégaphylle : surface foliaire supérieure à $20 \mathrm{dm}^{2}$.

Pour le calcul des différents spectres pondérés (SP) par abondance-dominance des formes biologiques retenues, la formule utilisée est la suivante :

\section{$\sum$ rec. moy. pour un trait biologique considéré}

$\mathrm{SP}=\frac{\sum \text { rec. moy. pour tous les traits biologiques identifiés }}{\sum \text { r. }}$

La correspondance entre les coefficients d'abondancedominance des espèces et leurs recouvrements moyens (rec. moy.) est indiquée dans le tableau I (BRAUn-BLANQUet, 1932).

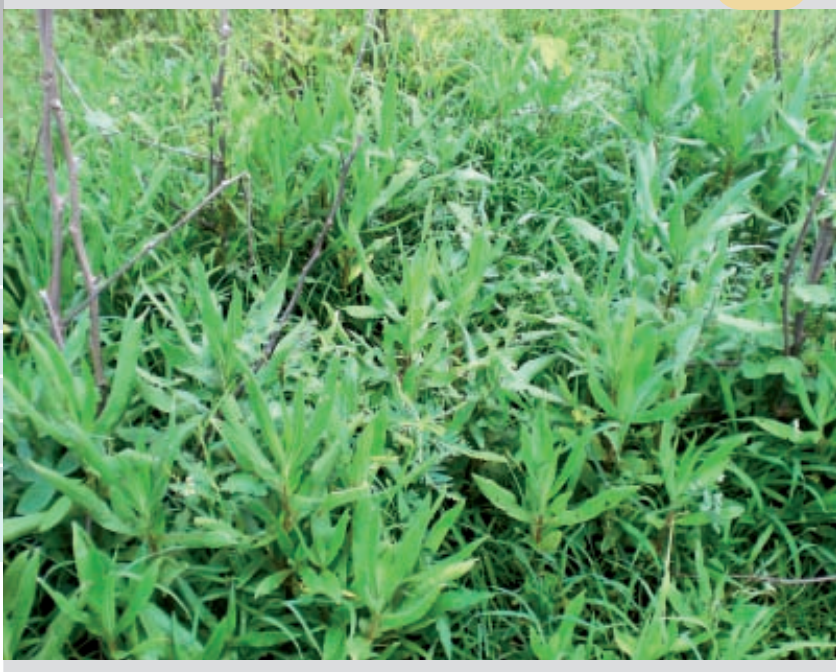

Faciès à Hygrophila auriculata dans les jeunes jachères de Rusizi (palmeraie). Photo F. Bangirinama.

\section{Définition d'indices successionnels.}

En début de succession se trouvent des espèces à majorité thérophytes à dispersion anémochore (BANGIRINAMA et al., 2009 b) dont les traits sont associés à la stratégie rudérale tolérante " $R$ » aux perturbations (GRIME, 1979) ; les espèces de fin de succession sont de type phanérophytes à dissémination zoochore développant des traits associés à la stratégie compétitrice « C ». L'évolution des types foliaires montre une augmentation progressive avec l'âge de la jachère des espèces mésophylles et macrophylles et une diminution d'espèces nanophylles, leptophylles et microphylles selon les stations (BANGIRINAMA et al., 2009 b).

Sur la base de ces conclusions et en vue d'illustrer la diversité fonctionnelle des jachères et de leur dynamique, nous avons retenu pour notre zone d'étude les formes qui semblent montrer des variations significatives entre les différents sites échantillonnés. Il s'agit des thérophytes et des phanérophytes pour les types biologiques, des espèces anémochores (sclérochores) et zoochores (sarcochores) pour les types des diaspores, des espèces microphylles et mésophylles pour les types foliaires.

\section{Indice succesionnel de forme de vie $\left(\mathrm{I}_{\mathrm{FV}}\right)$}

Cet indice représente, pour un site donné, le rapport entre le spectre pondéré des espèces phanérophytes et le spectre pondéré des espèces thérophytes. Il est donné par la formule suivante :

$$
\mathrm{I}_{\mathrm{FV}}=\frac{\sum \text { rec. moy. pour les phanérophytes }}{\sum \text { rec. moy. pour les thérophytes }}
$$


Indice successionnel de surface foliaire $\left(I_{\mathrm{SF}}\right)$

Cet indice représente le rapport entre le spectre pondéré des espèces mésophylles et le spectre pondéré des espèces microphylles. Il est donné par la formule suivante :

$\mathrm{I}_{\mathrm{SF}}=\frac{\sum \text { rec. moy. pour les espèces mésophylles }}{\sum \text { rec. moy. pour les espèces microphylles }}$

\section{Indice successionnel de dispersion $\left(I_{D}\right)$}

Cet indice représente le rapport entre le spectre pondéré des espèces zoochores sarcochores et le spectre pondéré des espèces anémochores sclérochores. Il est donné par la formule suivante :

\section{$I_{D}=\frac{\sum \text { rec. moy. pour les espèces sarcochores }}{\sum \text { rec. moy. pour les espèces sclérochores }}$}

\section{Analyses statistiques}

L'effet des facteurs site et âge de la jachère (stade) sur l'évolution temporelle des trois indices successionnels définis a été testé par une analyse de la variance (Anova) grâce au logiciel Statistica 6. Un ajustement polynomial d'ordre 2 a été réalisé sur le nuage des points de chaque indice successionnel. La significativité du coefficient de corrélation a été testée en appliquant la méthode de Pearson vu que le nombre d'observations était supérieur à dix. La valeur de $p$ du test de la pente nous a permis de décrire les trajectoires d'évolution temporelle de ces indices.

Tableau II.

Indices successionnels pour les différents sites échantillonnés.

\begin{tabular}{|c|c|c|c|c|}
\hline Site & Indice & Stade 1 & Stade 2 & Stade 3 \\
\hline \multirow[t]{3}{*}{ Ruvubu } & $\mathrm{I}_{\mathrm{FV}}$ & 0,43 & 0,71 & 2,31 \\
\hline & $\mathrm{I}_{\mathrm{SF}}$ & 0,14 & 0,21 & 0,5 \\
\hline & $I_{D}$ & 0,48 & 0,64 & 1,29 \\
\hline \multirow[t]{3}{*}{ Kigwena } & $\mathrm{I}_{\mathrm{FV}}$ & 0,65 & 1,67 & 4,11 \\
\hline & $\mathrm{I}_{\mathrm{SF}}$ & 0,5 & 0,44 & 0,65 \\
\hline & $I_{D}$ & 0,85 & 1,05 & 2,06 \\
\hline \multirow[t]{3}{*}{ Bururi } & $\mathrm{I}_{\mathrm{FV}}$ & 1,19 & 1,8 & 3,56 \\
\hline & $\mathrm{I}_{\mathrm{SF}}$ & 0,51 & 0,51 & 0,76 \\
\hline & $I_{D}$ & 0,92 & 1,5 & 1,55 \\
\hline \multirow[t]{3}{*}{ Rusizi (delta) } & $\mathrm{I}_{\mathrm{FV}}$ & 0,31 & 0,4 & 0,53 \\
\hline & $\mathrm{I}_{\mathrm{SF}}$ & 0,06 & 0,08 & 0,07 \\
\hline & $I_{D}$ & 0,38 & 0,54 & 1,4 \\
\hline \multirow[t]{3}{*}{ Rusizi (palmeraie) } & $\mathrm{I}_{\mathrm{FV}}$ & 0,29 & 0,44 & 1,47 \\
\hline & $\mathrm{I}_{\mathrm{SF}}$ & 0,03 & 0,03 & 0,07 \\
\hline & $I_{D}$ & 0,69 & 0,73 & 1,29 \\
\hline
\end{tabular}

\section{Résultats}

\section{Calculs d'indices successionnels}

L'analyse de nos résultats (tableau II) indique que ces trois indices définis augmentent avec l'âge de la jachère. Ils sont bas dans les jeunes jachères (stade 1) et élevés dans les jachères de plus de 5 ans (stade 3 ). Ces indices successionnels sont plus élevés dans les sites de Bururi et Kigwena et bas dans le site de Rusizi (delta).

\section{Évolution temporelle des indices successionnels}

\section{Indice successionnel de forme de vie}

L'ajustement polynomial d'ordre 2 de l'allure générale de l'indice successionnel de forme de vie (figure 2) donne un coefficient de corrélation de 0,61 avec un test de la pente significatif $(p<0,05)$. La croissance de cet indice est donc confirmée et est positivement corrélée à l'âge de la jachère. Il est en moyenne de $0,57 \pm 0,37,1,00 \pm 0,68$ et $2,47 \pm 1,47(n=5)$ respectivement dans les jeunes jachères, les jachères intermédiaires et les jachères de plus de 5 ans.

\section{Indice successionnel de surface foliaire}

L'ajustement de l'allure générale de l'évolution de l'indice successionnel de surface foliaire (figure 3 ) affiche un faible coefficient de corrélation $(r=0,37)$. Le test de la pente non significatif ( $p>0,05)$ corrobore cette situation. Il n'y a donc pas de relation entre l'évolution de cet indice et l'âge de la jachère. Il est en moyenne de $0,25 \pm 0,24,0,25 \pm 0,21$ et $0,41 \pm 0,32(n=5)$ respectivement dans les jeunes jachères, les jachères intermédiaires et les jachères de plus de 5 ans.

\section{Indice successionnel de dispersion}

L'indice successionnel de dispersion est positivement corrélé à l'âge de la jachère (figure 4) de manière significative $(r=0,68 ; p<0,05)$, ce qui prédit l'existence d'un effet significatif de l'âge de la jachère sur cet indice. Cet indice est en moyenne de $0,66 \pm 0,23,0,89 \pm 0,39$ et $1,52 \pm 0,32(n=5)$ respectivement dans les jeunes jachères, les jachères intermédiaires et les jachères de plus de 5 ans.

\section{Efficacité des indices successionnels dans la caractérisation du stade de la succession}

L'analyse de la variance a révélé des effets variables de l'âge de la jachère et du site sur les trois indices. L'effet de l'âge de la jachère (stade) est significatif $(p<0,05)$ sur les indices successionnels de forme de vie et de dispersion (tableau III) bien que la relation était faiblement perceptible (figures 2 à 4). Par contre, l'effet du site, non significatif sur les deux derniers indices, le devient sur l'in- 
Tableau III.

Résultats de l'analyse de la variance entre le stade,

le site et les indices successionnels de la dynamique post-culturale.

\begin{tabular}{l|c|l|l} 
Indice & Facteur & F-ratio & \multicolumn{1}{c}{$p$} \\
\hline $\mathrm{I}_{\mathrm{FV}}$ & Site & 1,60 & 0,248 \\
\hline & Stade & 4,93 & $0,027^{\star}$ \\
\hline $\mathrm{I}_{\mathrm{SF}}$ & Site & 13,87 & $0,000^{\star \star \star}$ \\
& Stade & 0,61 & 0,559 \\
\hline $\mathrm{I}$ & Site & 1,06 & 0,425 \\
\hline & Stade & 8,92 & $0,004^{\star *}$
\end{tabular}

$\mathrm{I}_{\mathrm{FV}}$ : Indice succesionnel de forme de vie ;

$\mathrm{I}_{\mathrm{SF}}$ : Indice successionnel de surface foliaire ;

$I_{D}$ : Indice successionnel de dispersion.

${ }^{\star}$ significatif au seuil de $5 \%$; ${ }^{\star *}$ significatif au seuil de $1 \%$;

*** significatif au seuil de $1 \%$.

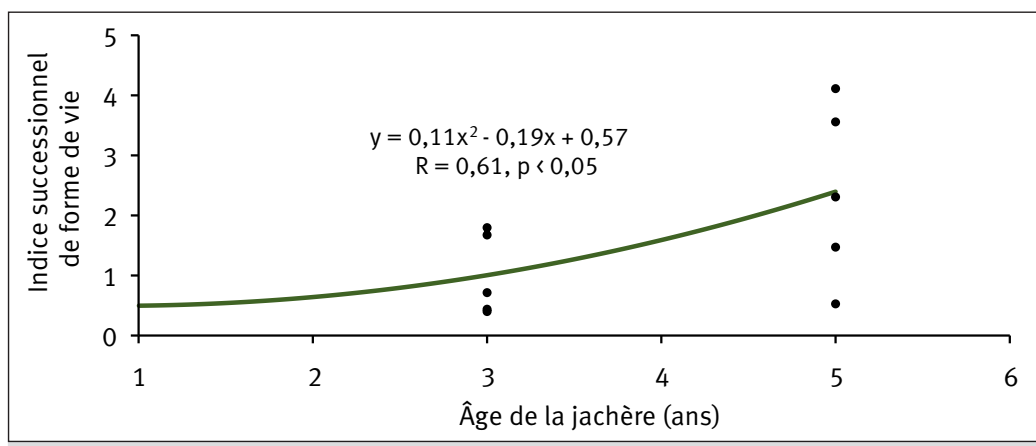

Figure 2.

Relation entre l'âge de la jachère et l'indice successionnel de forme de vie.

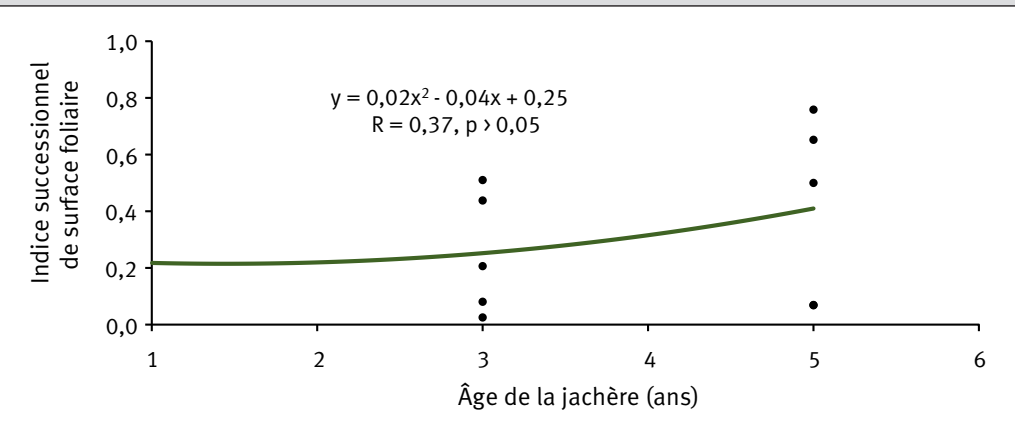

Figure 3.

Relation entre l'âge de la jachère et l'indice successionnel de surface foliaire.

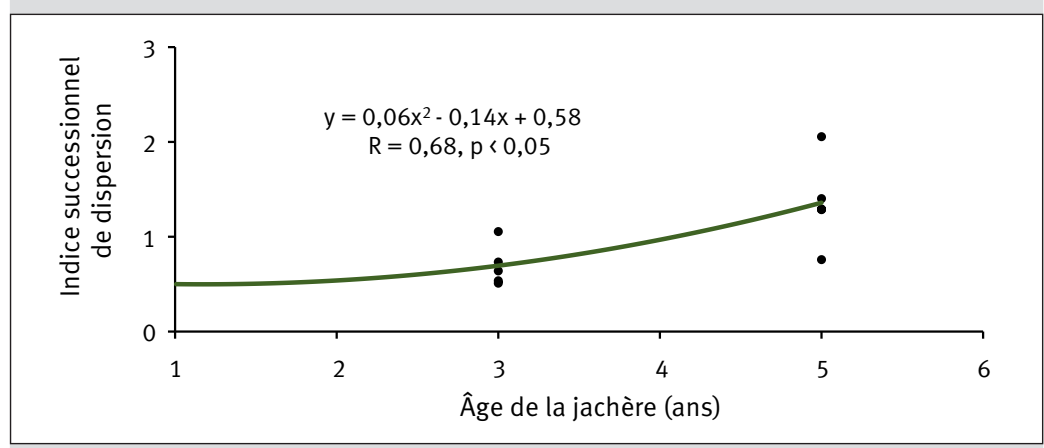

Figure 4.

Relation entre l'âge de la jachère et l'indice successionnel de dispersion. dice successionnel de surface foliaire (tableau III). II ressort ainsi que les indices successionnels de forme de vie et de dispersion constituent de bons outils de caractérisation du stade de la dynamique post-culturale.

\section{Trajectoires de la succession post-culturale}

\section{Indice successionnel de forme de vie}

L'évolution de l'indice successionnel de forme de vie pour les différents sites échantillonnés montre que la succession emprunte plusieurs trajectoires avec des vitesses très variables (tableau II). Les sites de Kigwena et Bururi affichent une tendance progressive de reconstitution des stades initiaux avec une dominance des phanérophytes. Le site de Kigwena présente la vitesse d'évolution la plus élevée. La vitesse d'évolution la plus lente est observée dans le site de Rusizi delta.

\section{Indice successionnel de surface foliaire}

En considérant l'indice successionnel de surface foliaire, on observe que les sites de Bururi, Kigwena et Ruvubu présentent une allure générale d'évolution positive (tableau II). Toutefois, le site de Kigwena semble connaître une évolution négative pendant la période de passage du stade 1 au stade 2. Les sites de Rusizi delta et Rusizi palmeraie ont, en revanche, une évolution presque constante.

\section{Indice successionnel de dispersion}

Les trajectoires suivies par les courbes d'évolution de l'indice de dispersion dans les différents sites sont également variables (tableau II). Les sites de Kigwena et de Bururi affichent une nette évolution progressive tendant vers la dominance des espèces forestières à diaspores zoochores. Dans les autres sites (Ruvubu, Rusizi palmeraie et Rusizi delta), les espèces anémochores persistent dans les stades avancés; en témoigne la faible croissance le long du gradient successionnel de l'indice de dispersion. 


\section{.}

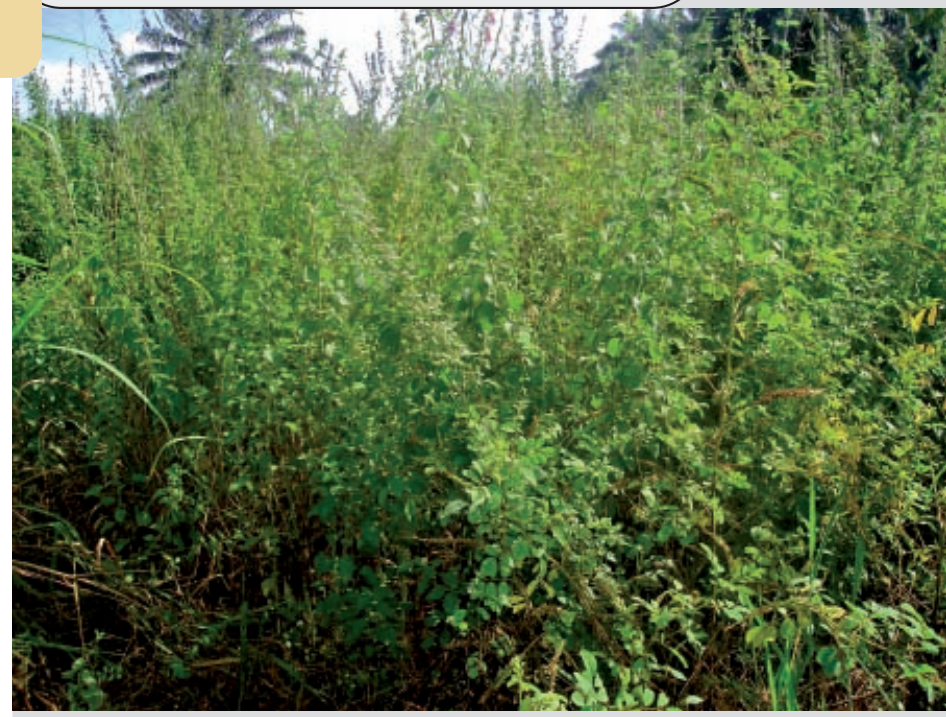

Faciès à Hyptis suaveolens dans les jeunes jachères de Kigwena.

Photo F. Bangirinama.



Faciès à Kotschya africana dans les jachères de plus de 5 ans à Bururi.

Photo F. Bangirinama.

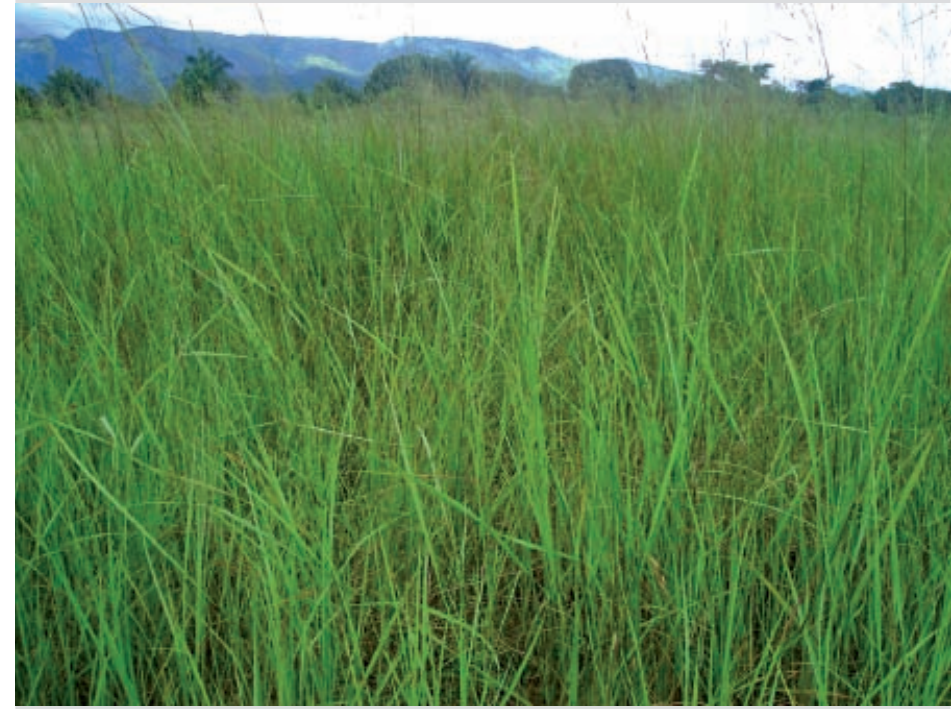

Faciès à Loudetia arundinacea dans les jachères de 3-5 ans à Kigwena.

Photo F. Bangirinama.

\section{Discussion}

\section{Pertinence des indices successionnels}

Plusieurs études (LEPART, EsCARrÉ, 1983 ; Fournier et al., 2001 ; BANGIRINAMA et al., 2009b) portant sur la succession post-culturale ont fourni plusieurs indicateurs permettant la caractérisation des différents stades de cette succession. Elles ont également inventorié les espèces indicatrices de chaque stade en fonction de la région. Cependant, ces informations manquent d'un caractère universel pour pouvoir être extrapolées. Les résultats de cette étude montrent que deux indices (indice successionnel de forme de vie et Indice successionnel de dispersion) présentent une corrélation significative avec le stade successionnel de la jachère. Leur pertinence dans la caractérisation de la dynamique post-culturale est donc confirmée.

L'efficacité de l'indice successionnel de surface foliaire n'est pas statistiquement confirmée dans cette étude. Au lieu de constituer un outil de caractérisation de la dynamique post-culturale, cet indice s'est révélé davantage lié aux caractéristiques du site.

Toutefois, les résultats ont mis en évidence des disparités régionales. Les régions plus arrosées (Bururi et Kigwena) avec environ 1500 mm par an de précipitations présentent des valeurs d'indices successionnels plus élevées que celles des régions soumises à une aridité de plus en plus intense (Rusizi delta et Rusizi palmeraie), avec moins de $1000 \mathrm{~mm}$ par an. Ce rôle du facteur climat dans le déterminisme du processus de la succession avait déjà été confirmé (LEPART, ESCARRÉ, 1983 ; FoURNIER et al., 2001 ; GRIME, 2001).

\section{Trajectoires de la succession post-culturale}

Un modèle de représentation des trajectoires d'évolution des différents faciès post-culturaux gouvernés par le gradient successionnel et les différentes pressions anthropiques a été développé par FourNiER et al. (2001). Les différents stades de la succession post-culturale apparaissent comme un remplacement temporel de plusieurs groupes fonctionnels de plantes depuis les thérophytes à diaspores anémochores vers les phanérophytes à diaspores zoochores. Nos résultats montrent que l'approche phytoécologique de l'évolution post-culturale permet de dégager les différentes trajectoires dynamiques. En l'absence de perturbation, les jachères connaissent un schéma général de recolonisation végétale tendant vers des formations arborées.

Cependant, ces phénomènes ne sont pas unidirectionnels. Les trajectoires suivies diffèrent d'une région à une autre. Dans cette étude, les régions humides (Bururi et Kigwena) tendent vers la formation d'écosystèmes forestiers. Le site de Ruvubu, à pluviosité moyenne, tend vers la formation d'écosystèmes dégradés (savanes arborées) tandis que les sites de la plaine de la Rusizi, hautement perturbés par les piétinements du bétail, connaissent une succession régressive. 


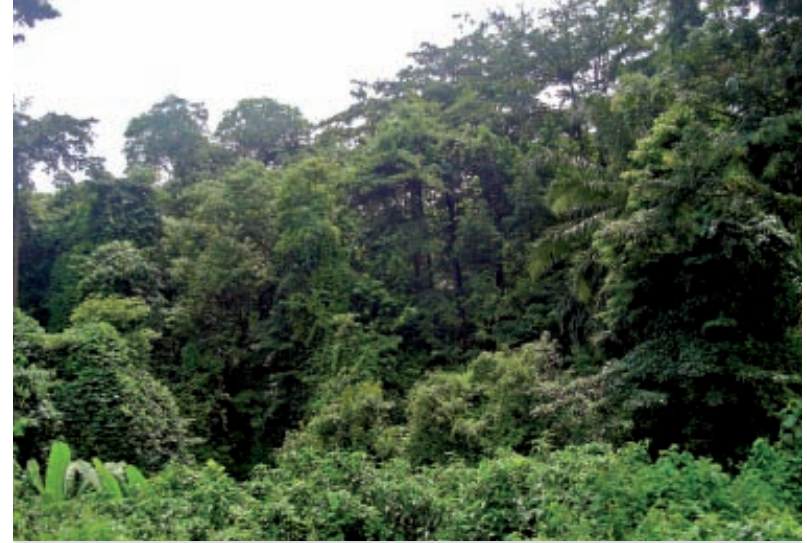

Forêt reconstituée à Pycnanthus angolensis à Kigwena. Photo F. Bangirinama.

Étudiant le gradient écoclimatique en Afrique occidentale, FOURNIER et al. (2001) ont formulé des conclusions similaires. Ces auteurs ont montré que la grande variété de physionomies et de compositions floristiques observée dans les jachères reflète les étapes du processus de succession post-culturale et leurs diverses modalités en fonction des milieux et des régions.

\section{Bilan : la restauration des écosystèmes initiaux}

Le déroulement de la succession post-culturale est très variable ; la durée même du processus de reconstitution n'est pas identique dans tous les cas. Si la subdivision de la dynamique post-culturale en trois stades (jeune jachère, jachère intermédiaire et jachère âgée) a été adoptée par d'autres auteurs (FouRniER et al., 2001), il reste évident que l'atteinte de ces stades peut se réaliser à des vitesses variables. Les compartiments dans un écosystème en dynamique ont chacun leur propre rythme d'évolution (ALARD et al., 1998).

Les résultats montrent que les régions humides (Bururi et Kigwena) connaissent une restauration de plus en plus rapide et tendent vers la reconstitution des formations forestières initiales, contrairement aux jachères de la plaine de la Rusizi. Le facteur le plus contraignant est différent pour chaque zone écoclimatique : sécheresse climatique et piétinement pour la plaine de la Rusizi et feux de brousse pour les savanes de la Ruvubu.

En fonction des contraintes principales, les étapes intermédiaires et l'état du stade le plus avancé adoptent des formes végétales possibles. En forêt humide, les stades herbacés sont plus ou moins tronqués et les herbacées pérennes totalement absentes ; la succession met surtout en jeu plusieurs vagues de ligneux. En savane, les stades herbacés sont bien développés, mais les ligneux, qui n'atteindront jamais des recouvrements très forts, sont seulement des pyrotolérants (GrouzIS, 1988). Quand l'aridité devient intense, les ligneux et les herbes pérennes sont peu abondants, et par conséquent certains stades caractéristiques du schéma général de la succession disparaissent.

\section{Remerciements}

Les auteurs remercient le Gouvernement du Burundi et l'Université libre de Bruxelles, respectivement pour la bourse et le complément de bourse octroyés à Frédéric Bangirinama dans le cadre de la réalisation de sa thèse doctorale.

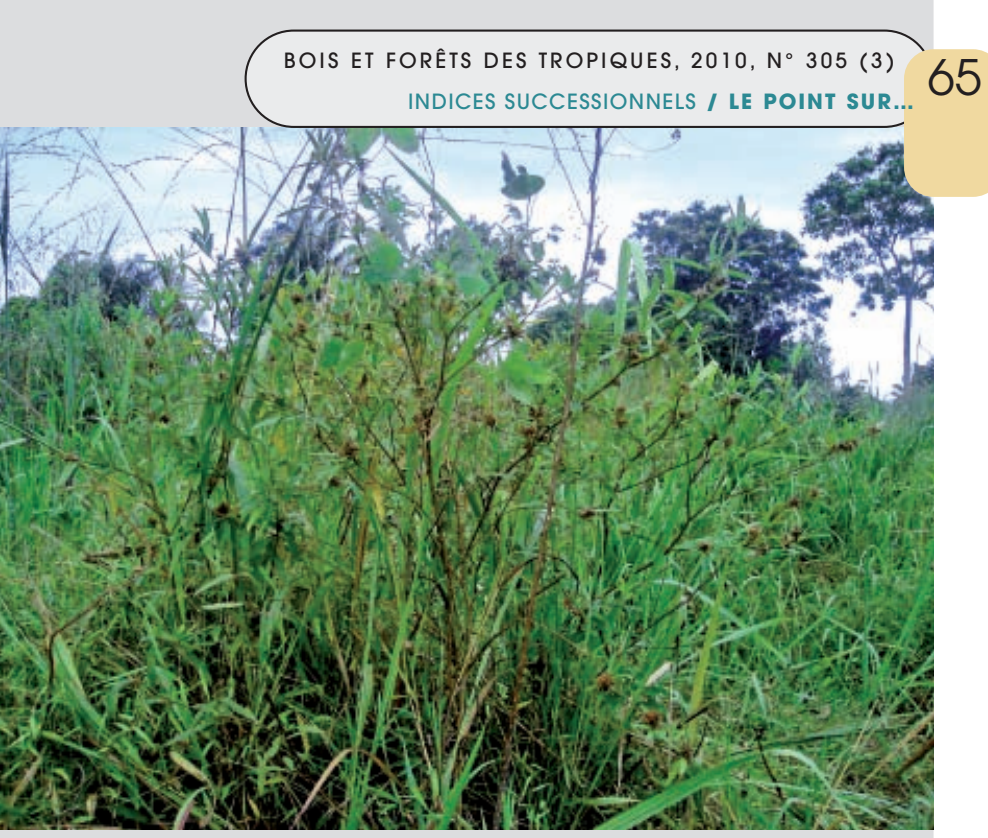

Faciès à Panicum maximum dans les jeunes jachères

de Kigwena.

Photo F. Bangirinama.

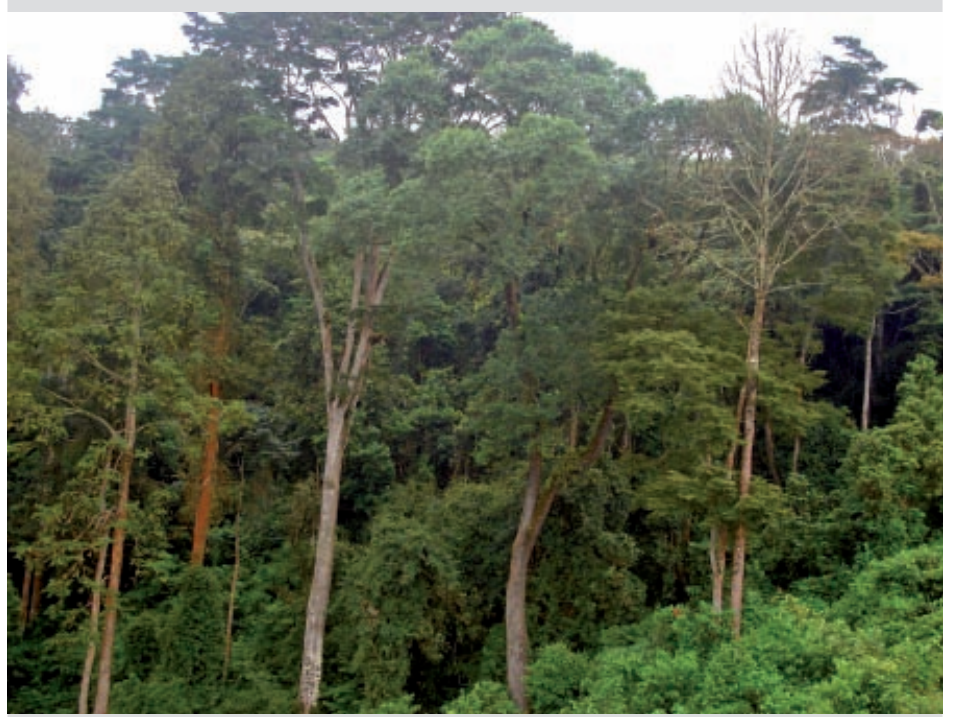

Forêt reconstituée à Entandrophragma excelsum à Bururi.

Photo F. Bangirinama.

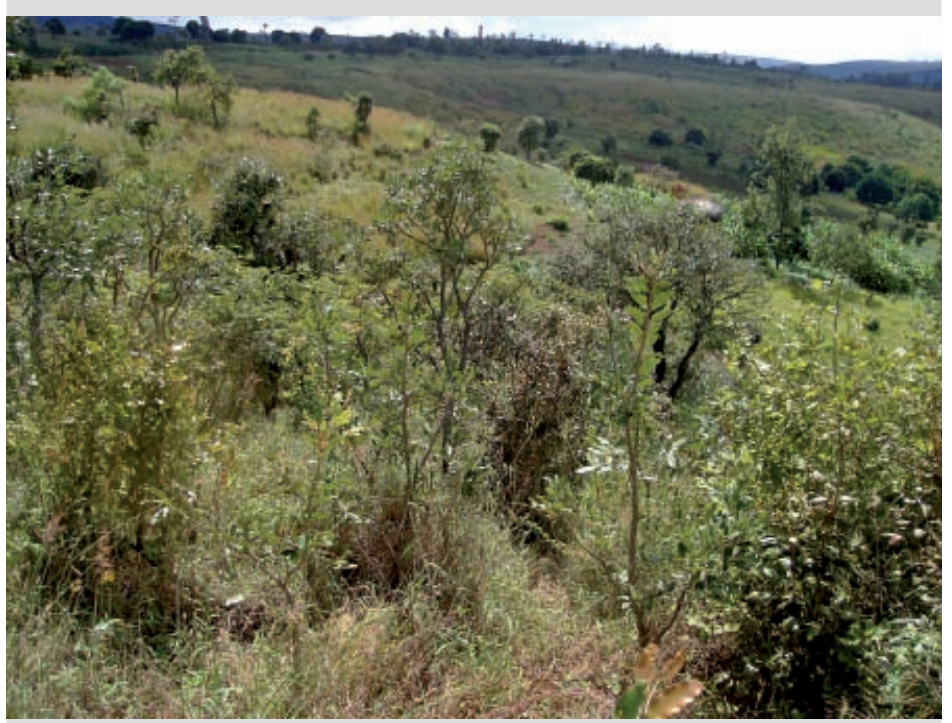

Stade à Parinari curatellifolia dans les jachères de plus de 5 ans à Ruvubu.

Photo F. Bangirinama. 


\section{Références bibliographiques}

ALARD D., POUDEVIGNE I., DUTOIT T., DECAENS T., 1998. Dynamique de la biodiversité dans un espace en mutation. Le cas des pelouses calcicoles de la basse vallée de Seine. Acta Fcologica, 19 : 275-284.

BANGIRINAMA F., BIGENDAKO M. J., LEJOLY J., 2009 a. Relations aire-espèces dans les jachères du périmètre de la réserve naturelle de Kigwena (Burundi). Acta Botanica Gallica, 156 (2) : 211-221.

BANGIRINAMA F., MASHARABU T., BIGENDAKO M. J., LEJOLY J., DE CANNIÈRE C., BOGAERT J., 2009 b. Évolution des paramètres floristiques au cours de la dynamique post-culturale dans les jachères du site Bibara dans le Parc National de la Ruvubu (Burundi). Bulletin Scientifique de l'Inecn, 7 : 3-13. BRAUN-BLANQUET J., 1932. Plant sociology. The study of plant communities. New York, Londres, Mac Gray Hill, 439 p.

CHAPIN F. S., 1993. Functional role of growth forms in ecosystem and global processes. In : Scaling physiological processes. Leaf to globe. San Diego, États-Unis, Academic Press, p. 287-312.

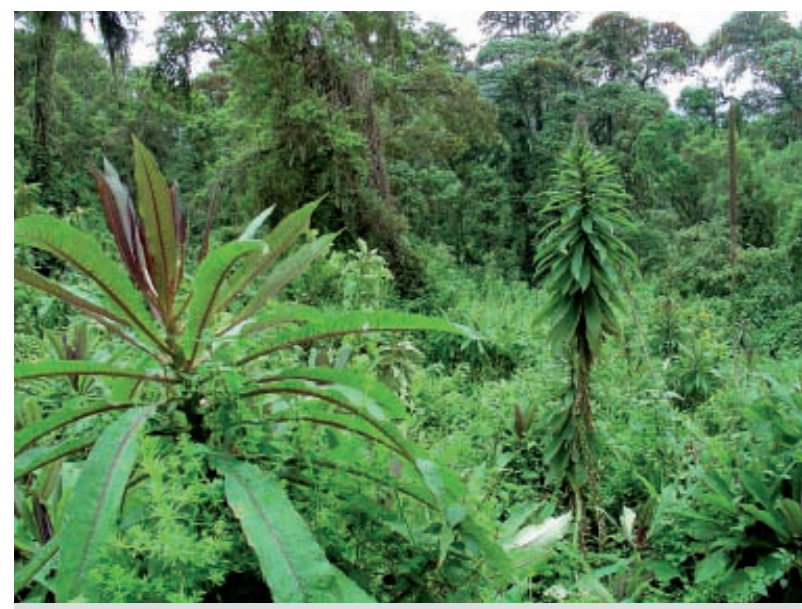

Stade à Lobelia giberroa dans les jachères de 3-5 ans à Bururi.

Photo F. Bangirinama.



Stade à Pteridium aquilinum et Virectaria major dans les jachères de plus de 5 ans à Bururi. Photo F. Bangirinama.
CORPEN, 2003. Des indicateurs pour des actions locales de maîtrise des pollutions de l'eau d'origine agricole : éléments méthodologiques, application aux produits phytosanitaires. Paris, France, Comité d'orientation pour des pratiques agricoles respectueuses de l'environnement, $136 \mathrm{p}$.

DANSEREAU P., LEMS K., 1957. The grading of dispersal types in plant communities. Contributions de l'Institut de Botanique de Montréal, 71 : 1-52.

FOURNIER A., FLORET C., GNAHOUA G. M., 2001. Végétation des jachères et succession post-culturale en Afrique tropicale. In : Floret C., Pontanier R. (éd.). La jachère en Afrique tropicale. II. De la jachère naturelle à la jachère améliorée : le point des connaissances. Paris, France, John Libbey Eurotext, p. $123-168$.

GRIME J. P., 1974. Vegetation classification by reference to strategies. Nature, $250: 26-31$.

GRIME J. P., 1979. Plant strategies and vegetation processes. New York, États-Unis, John Wiley and Sons, 222 p.

GRIME J. P., 2001. Plant strategies, vegetation processes, and ecosystem properties. Second edition. Chichester, Royaume-Uni, John Wiley and Sons, 456 p.

GROUZIS M., 1988. Structure, productivité et dynamique des systèmes écologiques sahéliens (mare d’Oursi, Burkina Faso). Paris, France, Orstom, coll. Études et thèses, 336 p.

LEBRUN J., 1947. La végétation de la plaine alluviale au sud du lac Édouard. Bruxelles, Belgique, Institut des parcs nationaux du Congo belge, coll. Exploration du Parc national Albert (Mission J. Lebrun 1937-1938), 800 p.

LEBRUN J.-P., STORK A. L., 1991-1997. Énumération des plantes à fleurs d'Afrique tropicale. Conservatoire et Jardin botaniques de la ville de Genève, Suisse, 4 volumes.

LEPART T. J., ESCARRÉ J., 1983. La succession végétale, mécanismes et modèles : analyse bibliographique. Bulletin d’Écologie, 14 : 133-178.

MAC ARTHUR R. H., WILSON E. O., 1967. The theory of island biogeography. Princeton, États-Unis, Princeton University Press.

RAUNKIAER C., 1934. The life's forms of plants and statistical plant geography. Londres, Royaume-Uni, Oxford University Press, $632 \mathrm{p}$.

SANTIAGO L., WRIGHT S., 2007. Leaf functional traits of tropical forest plants in relation to growth form. Functional Ecology, 21 (1) : 19-26.

TESTI B., 2008. Théophraste et Raunkiaer ont-ils pensé aux traits foliaires ? Master 1, Université des sciences et techniques du Languedoc, Centre d'écologie fonctionnelle et évolutive, Montpellier, France, 8 p.

WESTOBY M., FALSTER D. S., MOLES A. T., VESK P. A., WRIGHT J. J., 2002. Plant ecological strategies : some leading dimensions of variation between species. Annual Review of Ecology and Systematics, 33 : 125-159. 